\title{
Influences of Two Calculation Methods about Dynamic Tension on Vibration Characteristics of Cable-Bridge Coupling Model
}

\author{
Guangyun Min $\mathbb{D D}^{1}{ }^{1}$ Xiaohui Liu $\mathbb{D},{ }^{2}$ Chuan Wu $\mathbb{D},{ }^{3}$ Shuguang Yang $\mathbb{D},{ }^{1}$ and Mengqi Cai $\mathbb{i D}^{4}$ \\ ${ }^{1}$ School of Civil Engineering, Chongqing Jiaotong University, Chongqing 400074, China \\ ${ }^{2}$ State Key Laboratory of Mountain Bridge and Tunnel Engineering, Chongqing Jiaotong University, Chongqing 400074, China \\ ${ }^{3}$ State Grid Henan Electric Power Research Institute, Zhengzhou 450052, China \\ ${ }^{4}$ School of Architecture and Civil Engineering, Chengdu University, Chengdu 610106, China \\ Correspondence should be addressed to Xiaohui Liu; cqdxlxh@126.com
}

Received 12 November 2020; Revised 22 December 2020; Accepted 25 January 2021; Published 8 February 2021

Academic Editor: Li Li

Copyright (c) 2021 Guangyun Min et al. This is an open access article distributed under the Creative Commons Attribution License, which permits unrestricted use, distribution, and reproduction in any medium, provided the original work is properly cited.

\begin{abstract}
For the cable-bridge coupling model, the dynamic tension of cables is an important parameter to study the vibration characteristics of the model. Based on this concept, two calculation methods about dynamic tension of cables were introduced in great detail, and the influences of these two calculation methods on the vibration characteristics of cable-bridge coupling model were systematically investigated. Firstly, the vibration equation of the cable was derived based on the variational principle for Hamiltonian, and the vibration equation of the bridge deck was obtained by Newton's law. Then, the vibration equation of the cable and bridge deck was transformed into ordinary differential vibration equation by the Galerkin method. In addition, the differences of the coefficients in the ordinary differential vibration equation obtained by these two calculation methods about dynamic tension were compared, and a parameter analysis was listed. Finally, the resonance mode of the cablebridge coupling model was analyzed by a multiple scales method, and an example analysis was listed. The results of parameter analysis show that there are obvious differences in the linear coefficient and nonlinear coefficient of the ordinary differential vibration equation obtained by these two calculation methods. The results of example analysis show that, for the cable-bridge coupling model with 1:1 resonance, the amplitude of the model would not be different because of the two calculation methods about dynamic tension, but the amplitude of the cable would be affected by the calculation method significantly. It can be found that the research conclusions here can be helpful to the perfection of theoretical modeling and has certain guiding value for practical engineering.
\end{abstract}

\section{Introduction}

Because cables have the characteristics of light-weight, largeflexibility, and small-damping, those are used in civil engineering, navigation engineering, aerospace engineering, and other fields usually [1-4]. In civil engineering, from the investigation of long-span stay-cable bridges, it can find that large-scale vibration of stay-cables may occur under the loads of winds, rains, pedestrians, or vehicles [5-7]. In addition, the vibration characteristics of stay-cables are also affected by the tower column and bridge deck, which would make the dynamic behavior of stay-cables be more complex.
For example, when the vibration frequency of stay-cables is proportional to the vibration frequency of the bridge deck, the resonance would occur in the cable-bridge coupling system. And in this condition, even small disturbances would also let stay-cables generate large displacement.

Scholars have made lots of contributions to the study of the vibration characteristics of the cable-bridge coupling model. In 1998, the authors in [8] established the cablebridge coupling model and the vibration equation of it is derived. The research results showed that, under certain conditions, the stay-cables may resonate with the bridge deck, which would bring adverse effects to the safety of the 
bridge. In 2001, the parametric vibration model of staycables was established in [9], and the vibration characteristics of stay-cables were analyzed. Then, it proposed to apply dampers on the bridge deck to control the large-amplitude vibration of stay-cables. In 2003, the cable-bridge coupling model was established in [10], and the displacement response of stay-cables was obtained by the fourth-order Runge-Kutta method. The research results showed that the vibration characteristics of stay-cables and bridge deck are related to the sag and inclination of the stay-cables. In [11], the coupling model of bridge deck and double-cables is established, and the interaction between one cable and the other cable is further considered. The research results showed that, by changing the parameters of one cable, the vibration characteristics of another cable would be greatly affected; that is, the coupling interaction between cable and cable is significant. In 2012, the continuous nonlinear refined vibration model of tower-cable-bridge deck was established in [12], and the influences of frequency ratio of the bridge deck to cables, amplitude of excitation, force of cables, and damping on the vibration characteristics of the model were studied. In 2016, considering the synergistic effects of cable-tower-beam, the authors in [13] analyzed the parameter vibration mechanism of cables and calculated the dynamic characteristics of the bridge deck and cables. The research results can provide a theoretical value for the installation of vibration suppression devices for cables. In 2017, Zhao et al. [14] were concerned with the temperature effect on the vibration of a cable-stayed beam, the research results showed that the effect of temperature plays a dominant role on the vibration characteristics of the cable-stayed beam, and the effect is closely related with the initial tension force and the stiffness ratio.

For investigating the vibration characteristics of arbitrary vibration models, such as cables $[15,16]$, bridges $[17,18]$, iced conductors $[19,20]$, and plates [21-24], the partial differential vibration equation of those vibration models should be established firstly. As known for us, for the cable-bridge coupling model, the dynamic tension of cables is an important parameter to study the vibration characteristics of the model. Based on this concept, two calculation methods about dynamic tension of cables were presented in this paper. Under the two calculation methods about dynamic tension of cables, the partial differential vibration equation of the model can be converted into ordinary differential vibration equation by two methods. One is to average the dynamic tension of cables over the span length of cables, and then, the Galerkin method is used to convert the partial differential vibration equation into ordinary differential vibration equation directly. The other is not to average the dynamic tension of cables over the span length, and then, the Galerkin method is used. There are different calculation methods about dynamic tension of cables, the partial differential vibration equation of the cablebridge coupling model would be different, and then, the ordinary differential vibration equation of the model would also be different. Since the two calculation methods about dynamic tension both are reasonable and have their own advantages, whether these two calculation methods about dynamic tension would affect the coefficients of vibration equation of the cable-bridge coupling model or lead to the vibration characteristics of the model to be different has not been investigated by scholars.

Based on these conclusions above, the influences of the two calculation methods about dynamic tension on the vibration characteristics of a cable-bridge coupling model has been systematically studied in this paper. Firstly, the cable-bridge coupling model is established, and the vibration equation of the model is derived in this paper. Second, the differences of the coefficients in the ordinary differential equation obtained by the two calculation methods of dynamic tension were compared. Furthermore, the resonance mode of the cable-bridge coupling model is analyzed by the multiple scales method. Finally, the influence of the two calculation methods of dynamic tension on the vibration characteristics of cable-bridge coupling model is further analyzed. The research results of this paper can be good for improving the theoretical modeling and offering some reference values to the actual engineering.

\section{Vibration Equation}

In order to facilitate the study and reflect the essence of the research problem in this paper, the following assumptions are made:

(1) The bending stiffness, torsional stiffness, and shear stiffness of the cable are not considered.

(2) The static equilibrium configuration of the cable is represented by parabola.

(3) The constitutive relation of the cable satisfies Hooke's law.

In this paper, the bridge deck is simplified as an equivalent mass block with certain stiffness. And, based on this concept, the cable-bridge coupling model can be established, which is shown in Figure 1.

In Figure 1,y is the static equilibrium configuration of the cable, $\xi$ is the dynamic displacement of the cable deviating from the static equilibrium configuration, $\theta$ is the angle between the cable and the horizontal direction, and $X$ is the displacement of the bridge deck.

According to [20], it can be obtained that the vibration of the cable would mainly occur in the $y$-axis direction (inplane), so this paper would only consider the vibration of the cable in the $y$-axis direction (in-plane). Based on the variational principle for Hamiltonian, the following results can be obtained:

$$
\int_{t_{1}}^{t_{2}}\left(\delta K^{V}-\delta \prod+\delta W\right) \mathrm{d} t=0,
$$

where $K^{V}$ represents the kinetic energy of the cable, $\Pi$ represents the potential energy of the cable, $W$ represents the work done by the non-conservative forces, $t$ represents the time, $t_{1}$ is the initial time, and $t_{2}$ is the final time.

The items in equation (1) are as follows: 


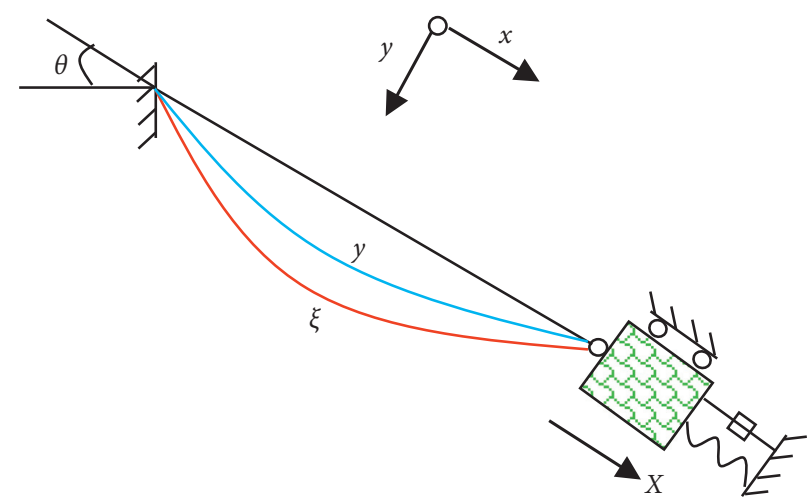

Figure 1: Cable-bridge coupling model.

$$
\begin{aligned}
K^{V} & =\frac{1}{2} \int_{0}^{L} m\left(\frac{\partial \xi}{\partial t}\right)^{2} \mathrm{~d} x \\
\prod & =\int_{0}^{L}\left[H h+\frac{1}{2} E A h^{2}\right] \mathrm{d} x ; \\
W & =\int_{0}^{L}\left(m g \cos \theta+\varsigma_{C} \frac{\partial \xi}{\partial t}\right) \mathrm{d} x,
\end{aligned}
$$

where $H$ represents the initial static tension, $E A$ represents the tensile stiffness, $h$ is the dynamic tension, $m$ is the unit mass of the cable, $\varsigma_{C}$ is the damping coefficient of the cable, $L$ is the span length of the cable, and $g$ is the acceleration of gravity.

By substituting equations (2) into (1), the in-plane vibration equation of the cable can be obtained as follows:

$$
(H+h) \frac{\partial^{2} \xi}{\partial x^{2}}+h \frac{\mathrm{d}^{2} y}{\mathrm{~d} x^{2}}=m \frac{\partial^{2} \xi}{\partial t^{2}}+\varsigma_{C} \frac{\partial \xi}{\partial t} .
$$

According to Newton's law, the vibration equation of bridge deck is

$$
M \frac{\partial^{2} X}{\partial t}+C \frac{\partial X}{\partial t}+K X+h=0
$$

where $K$ is the equivalent stiffness of the bridge deck, $C$ is the viscous damping of the bridge deck, and $M$ is the equivalent mass of the bridge deck.

The vibration equation of the cable-bridge coupling model can be obtained by combining equations (3) and (4), which is

$$
\left\{\begin{array}{l}
(H+h) \frac{\partial^{2} \xi}{\partial x^{2}}+h \frac{\mathrm{d}^{2} y}{\mathrm{~d} x^{2}}=m \frac{\partial^{2} \xi}{\partial t^{2}}+\varsigma_{C} \frac{\partial \xi}{\partial t} \\
M \frac{\partial^{2} X}{\partial t}+C \frac{\partial X}{\partial t}+K X+h=0 .
\end{array}\right.
$$

\section{Two Calculation Methods}

3.1. The First Calculation Method. In order to facilitate the analysis about vibration characteristics of the cable-bridge coupling model, the calculation equation of dynamic tension about the cable should be obtained firstly. The microelement length $\mathrm{d} s$ on the static equilibrium configuration of the cable and the microelement length $\mathrm{d} s$ ' on the dynamic equilibrium configuration of the cable are taken for the research object, respectively (see Figure 2 for details).

The cable can be regarded as a continuum, and then, the calculation equation about dynamic tension of the cable can be obtained according to the continuum theory, which is

$$
\begin{aligned}
h & =E A e(x)=\frac{\left(\mathrm{d} s^{\prime}+\mathrm{d} s\right)\left(\mathrm{d} s^{\prime}-\mathrm{d} s\right)}{\mathrm{d} s\left(\mathrm{~d} s^{\prime}+\mathrm{d} s\right)} \\
& \approx \frac{\left(\mathrm{d} s^{\prime}\right)^{2}-(\mathrm{d} s)^{2}}{2(\mathrm{~d} s)^{2}} \approx E A\left[\frac{\mathrm{d} y}{\mathrm{~d} x} \frac{\partial \xi}{\partial x}+\frac{1}{2}\left(\frac{\partial \xi}{\partial x}\right)^{2}\right]
\end{aligned}
$$

where $e(x)$ is the strain.

Equation (6) is the dynamic tension caused by the change in the configuration of the cable, and the change in dynamic tension caused by the vibration of bridge deck is not considered. If the influence of the vibration of bridge deck on dynamic tension is considered, equation (2) can be rewritten as follows:

$$
h_{1}=E A \varepsilon \approx E A\left\{\left[\frac{\mathrm{d} y}{\mathrm{~d} x} \frac{\partial \xi}{\partial x}+\frac{1}{2}\left(\frac{\partial \xi}{\partial x}\right)^{2}\right]+\frac{X}{L}\right\} .
$$

Equation (7) is the first calculation method of dynamic tension.

By substituting equation (7) into (5), the vibration equation of cable-bridge coupling model under the first calculation method about dynamic tension can be obtained, which is 


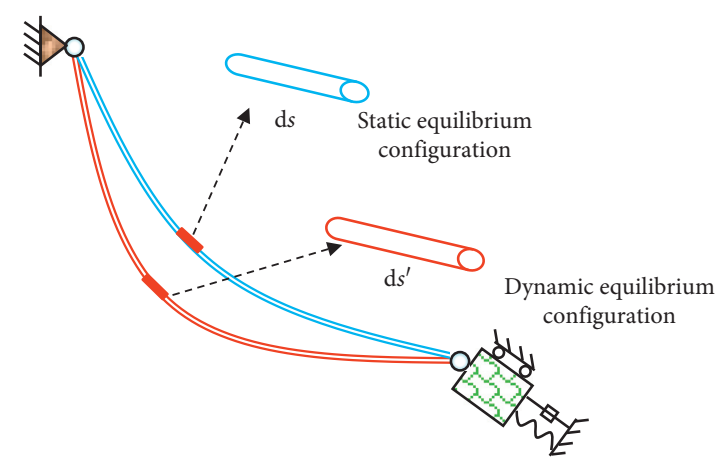

FIgURE 2: Microelement diagram of cable.

$$
\left\{\begin{array}{l}
\left(H+E A\left\{\left[\frac{\mathrm{d} y}{\mathrm{~d} x} \frac{\partial \xi}{\partial x}+\frac{1}{2}\left(\frac{\partial \xi}{\partial x}\right)^{2}\right]+\frac{X}{L}\right\}\right) \frac{\partial^{2} \xi}{\partial x^{2}}+E A\left\{\left[\frac{\mathrm{d} y}{\mathrm{~d} x} \frac{\partial \xi}{\partial x}+\frac{1}{2}\left(\frac{\partial \xi}{\partial x}\right)^{2}\right]+\frac{X}{L}\right\} \frac{\mathrm{d}^{2} y}{\mathrm{~d} x^{2}}=m \frac{\partial^{2} \xi}{\partial t^{2}}+\varsigma_{C} \frac{\partial \xi}{\partial t} \\
M \frac{\partial^{2} X}{\partial t}+C \frac{\partial X}{\partial t}+K X+E A\left\{\left[\frac{\mathrm{d} y}{\mathrm{~d} x} \frac{\partial \xi}{\partial x}+\frac{1}{2}\left(\frac{\partial \xi}{\partial x}\right)^{2}\right]+\frac{X}{L}\right\}=0 .
\end{array}\right.
$$

3.2. The Second Calculation Method. In practical engineering, the axial vibration of the cable is far less than its transverse vibration; that is to say, the effect of axial inertia force of the cable can be ignored. Based on this concept, equation (7) can be averaged over the range of 0 to $L$; that is, equation (7) can be rewritten as follows:

$$
h_{2}=E A \varepsilon \approx \frac{E A}{L}\left\{\int_{0}^{L}\left[\frac{\mathrm{d} y}{\mathrm{~d} x} \frac{\partial \xi}{\partial x}+\frac{1}{2}\left(\frac{\partial \xi}{\partial x}\right)^{2}\right] \mathrm{d} x+X\right\} .
$$

Equation (9) is the second calculation method of dynamic tension.

By substituting equation (9) into (5), the vibration equation of the cable-bridge coupling model under the second calculation method about dynamic tension can be obtained, which is

$$
\left\{\begin{array}{l}
\left(H+\frac{E A}{L}\left\{\int_{0}^{L}\left[\frac{\mathrm{d} y}{\mathrm{~d} x} \frac{\partial \xi}{\partial x}+\frac{1}{2}\left(\frac{\partial \xi}{\partial x}\right)^{2}\right] \mathrm{d} x+X\right\}\right) \frac{\partial^{2} \xi}{\partial x^{2}}+\frac{E A}{L}\left\{\int_{0}^{L}\left[\frac{\mathrm{d} y}{\mathrm{~d} x} \frac{\partial \xi}{\partial x}+\frac{1}{2}\left(\frac{\partial \xi}{\partial x}\right)^{2}\right] \mathrm{d} x+X\right\} \frac{\mathrm{d}^{2} y}{\mathrm{~d} x^{2}}=m \frac{\partial^{2} \xi}{\partial t^{2}}+\varsigma_{C} \frac{\partial \xi}{\partial t^{\prime}}, \\
M \frac{\partial^{2} X}{\partial t}+C \frac{\partial X}{\partial t}+K X+\frac{E A}{L}\left\{\int_{0}^{L}\left[\frac{\mathrm{d} y}{\mathrm{~d} x} \frac{\partial \xi}{\partial x}+\frac{1}{2}\left(\frac{\partial \xi}{\partial x}\right)^{2}\right] \mathrm{d} x+X\right\}=0 .
\end{array}\right.
$$

3.3. Differences in Coefficients. According to [20], the vibration characteristics of the cable are mainly affected by the first-order mode shape. Therefore, the first-order mode truncation method is used here, and the influences of higherorder mode shape on the vibration behaviors of the cable would be considered in subsequent research.

According to the first-order mode truncation method, the dynamic displacement $(\xi)$ of the cable can be written as the product of time function and mode shape function, which is

$$
\xi=q(t) \phi(x)
$$

By substituting equation (11) into (8), based on the Galerkin method, the ordinary differential vibration equation of the cable-bridge coupling model under the first calculation method about dynamic tension can be obtained:

$$
\left\{\begin{array}{l}
v_{1} \ddot{q}(t)+v_{2} \dot{q}(t)+v_{3} q(t)+v_{4} q^{2}(t)+v_{5} q^{3}(t)+v_{6} X+v_{7} X q(t)=0, \\
v_{8} \ddot{X}(t)+v_{9} \dot{X}(t)+v_{10} X(t)+v_{11} q(t)+v_{12} q^{2}(t)=0,
\end{array}\right.
$$

where the dot represents the derivative of $t$ and the calculation equations of coefficients involved in equation (12) are as follows: 


$$
\begin{aligned}
& \nu_{1}=-\int_{0}^{L} m \phi^{2}(x) \mathrm{d} x \\
& v_{2}=-\int_{0}^{L} \varsigma_{C} \phi^{2}(x) \mathrm{d} x \\
& \nu_{3}=\int_{0}^{L} H \phi^{\prime \prime}(x) \phi(x) \mathrm{d} x+\int_{0}^{L} E A y^{\prime} y^{\prime \prime} \phi^{\prime}(x) \phi(x) \mathrm{d} x \\
& v_{4}=\int_{0}^{L} E A y^{\prime} \phi^{\prime}(x) \phi(x) \phi(x) \mathrm{d} x+\int_{0}^{L} \frac{E A}{2} y^{\prime \prime} \phi^{\prime 2}(x) \phi(x) \mathrm{d} x \\
& v_{5}=\int_{0}^{L} \frac{E A}{2} \phi^{\prime 2}(x) \phi^{\prime \prime}(x) \phi(x) \mathrm{d} x \\
& v_{6}=\frac{E A}{L} \int_{0}^{L} y^{\prime \prime} \phi(x) \mathrm{d} x ; \\
& \nu_{7}=\frac{E A}{L} \int_{0}^{L} \phi^{\prime \prime}(x) \phi(x) \mathrm{d} x ; \\
& v_{8}=\int_{0}^{L} M \phi(x) \mathrm{d} x \\
& v_{9}=\int_{0}^{L} C \phi(x) \mathrm{d} x \\
& v_{10}=\int_{0}^{L} \frac{E A}{L} \phi(x) \mathrm{d} x+\int_{0}^{L} K \phi(x) \mathrm{d} x ; \\
& v_{11}=\int_{0}^{L} E A y^{\prime} \phi^{\prime}(x) \phi(x) \mathrm{d} x ; \\
& v_{12}=\int_{0}^{L} \frac{E A}{2} \phi^{\prime 2}(x) \phi(x) \mathrm{d} x
\end{aligned}
$$

In the same way, by substituting equation (11) into (10), based on the Galerkin method, the ordinary differential vibration equation of the cable-bridge coupling model under the second calculation method about dynamic tension can be obtained:

$$
\left\{\begin{array}{l}
\vartheta_{1} \ddot{q}(t)+\vartheta_{2} \dot{q}(t)+\theta_{3} q(t)+\vartheta_{4} q^{2}(t)+\vartheta_{5} q^{3}(t) \\
\quad+\vartheta_{6} X+\vartheta_{7} X q(t)=0 \\
\vartheta_{8} \ddot{X}(t)+\vartheta_{9} \dot{X}(t)+\vartheta_{10} X(t)+\vartheta_{11} q(t)+\vartheta_{12} q^{2}(t)=0 .
\end{array}\right.
$$

The calculation equations of coefficients involved in equation (14) are as follows:

$$
\begin{aligned}
& \vartheta_{1}=-\int_{0}^{L} m \phi^{2}(x) \mathrm{d} x ; \\
& \vartheta_{2}=-\int_{0}^{L} \varsigma_{C} \phi^{2}(x) \mathrm{d} x ; \\
& \vartheta_{3}= \int_{0}^{L} H \phi^{\prime \prime}(x) \phi(x) \mathrm{d} x \\
&+\int_{0}^{L} \frac{E A}{L} \int_{0}^{L} y^{\prime} \phi^{\prime}(x) \mathrm{d} x y^{\prime \prime} \phi(x) \mathrm{d} x ; \\
& \vartheta_{4}= \int_{0}^{L} \frac{E A}{L} \int_{0}^{L} y^{\prime} \phi^{\prime}(x) \mathrm{d} x \phi(x)^{\prime \prime} \phi(x) \mathrm{d} x \\
& \vartheta_{11}=+\int_{0}^{L} \frac{E A}{2 L} \int_{0}^{L} \phi^{\prime 2}(x) \mathrm{d} x y^{\prime \prime} \phi(x) \mathrm{d} x ; \\
& \vartheta_{9}^{L} \frac{E A}{L L} \phi^{\prime 2}(x) \mathrm{d} x \phi(x) \mathrm{d} x . \\
& \vartheta_{5}= \int_{0}^{L} \frac{E A}{2 L} \int_{0}^{L} \phi^{\prime 2}(x) \mathrm{d} x \phi^{\prime \prime}(x) \phi(x) \mathrm{d} x ; \\
& \vartheta_{7}= \frac{E A}{L} \int_{0}^{L} \int_{0}^{L} \phi^{\prime \prime}(x) \phi(x) \mathrm{d} x ; \\
& \vartheta^{\prime \prime} \phi(x) \mathrm{d} x ; \int_{0}^{L} \phi(x) \mathrm{d} x ;
\end{aligned}
$$

By comparing equations (13) and (15), it can be found that the coefficients $\left(\nu_{3}\right.$ and $\vartheta_{3} ; \nu_{4}$ and $\vartheta_{4} ; \nu_{5}$ and $\vartheta_{5} ; \nu_{11}$ and $\vartheta_{11} ; \nu_{12}$ and $\vartheta_{12}$ ) in the ordinary differential vibration equation of cable-bridge coupling model obtained by the first calculation method about dynamic tension and the second calculation method about dynamic tension have obvious differences. The differences of these coefficients may lead to the differences in vibration characteristics of the cable-bridge coupling model, and the differences between them would also lead to different natural frequencies of the model. Therefore, from the analysis here, it can also be found that it is necessary to consider the two calculation methods about dynamic tension when studying the vibration characteristics of cable-bridge coupling model. 
3.4. Parameter Analysis. From the analysis form in the section above, it can be seen that there are some differences in some coefficients of the ordinary differential vibration equation obtained by the two calculation methods about dynamic tension. The differences of some coefficients in vibration equation of cable-bridge coupling model under the two calculation methods about dynamic tension are studied systematically based on the physical parameters in [11]. Also, based on Matlab, the curves about influences of span length on coefficients are obtained, which is shown in Figure 3.

It can be seen from Figure 3 that, with the increasing $L$, the coefficients $\left(\nu_{4}\right.$ and $\vartheta_{4}, \nu_{5}$ and $\vartheta_{5}$, and $\nu_{11}$ and $\left.\vartheta_{11}\right)$ would increase; with the increasing $L$, the coefficients $\left(\nu_{3}\right.$ and $\vartheta_{3}$ and $\nu_{12}$ and $\vartheta_{12}$ ) would decrease; therefore, the coefficients obtained by the two calculation methods have the same variation law; that is, these two calculation methods would not affect the variation law of the coefficients. It can also be obtained from Figure 3 that when $L$ is greater than $300 \mathrm{~m}$, the ratio of coefficients obtained by these two calculation methods is a certain value; that is, different calculation methods of dynamic tension would affect the value of coefficients; when $L$ is less than $300 \mathrm{~m}$, the ratio of coefficients obtained by the two methods is a variable. Moreover, with the increasing $L$, the ratio of coefficients would increase, and finally, the ratio would tend to be constant; these two calculation methods would also affect the vibration frequency of the cable, which may lead to the vibration characteristics of the cable-bridge coupling model to be different. Therefore, it has certain theoretical significances and engineering values to study the influence of these two different calculation methods about dynamic tension on the vibration characteristics of the cable-bridge coupling model. In the following section, the influence of these two calculation methods about dynamic tension on the internal resonance of the cablebridge coupling model would also be studied based on a numerical example.

\section{Multiple Scales Method}

In practical engineering, when the frequency of the cable is proportional to the frequency of the bridge deck, it would let the cable-bridge coupling system generate resonance. The resonance would greatly let the amplitude of the cable and bridge deck be much bigger, which would damage the stability of structure and threaten the safety of personal. Therefore, it is necessary to analyze the resonance mode of the cable-bridge coupling system. In recent decades, many effective methods have been proposed to solve the nonlinear vibration equation. The multiple scales method is a common method to solve the nonlinear vibration equation and is one of the effective methods to analyze the nonlinear resonance mode. Based on this concept, the multiple scales method is used to solve the resonance mode of the cable-bridge coupling system here.

From equations (14) and (12), it can be obtained that the form of vibration equation under the two calculation methods of dynamic tension is the same even though the coefficients of some items in the vibration equation are different. For the form of the vibration equation is exactly the same, only the solution process of resonance mode of the cable-bridge coupling system under the first calculation method of dynamic tension is listed as shown in the following section. And the resonance mode of the cable-bridge coupling system under the second calculation method of dynamic tension can be deduced by analogy.

In order to satisfy the solution form of multiple scales method, equation (12) should be rewritten as follows:

$$
\left\{\begin{array}{l}
\ddot{Q}_{1}+\omega_{\mathrm{Q} 1}^{2} Q_{1}=\varepsilon\left(\xi_{\mathrm{Q} 1} \dot{Q}_{1}+\xi_{\mathrm{Q} 2} Q_{1}^{2}+\xi_{\mathrm{Q} 3} Q_{1}^{3}+\xi_{\mathrm{Q} 4} Q_{2}+\xi_{\mathrm{Q} 5} Q_{1} Q_{2}\right), \\
\ddot{Q}_{2}+\omega_{\mathrm{Q} 2}^{2} Q_{2}=\varepsilon\left(\xi_{\mathrm{Q} 6} \dot{Q}_{2}+\xi_{\mathrm{Q} 7} Q_{1}+\xi_{\mathrm{Q} 8} Q_{1}^{2}\right),
\end{array}\right.
$$

where $Q_{1}$ is $q(t), Q_{2}$ is $X(t)$, and $\varepsilon$ is a dimensionless small parameter. The calculation formulas of other coefficients are as follows:

$$
\begin{aligned}
& \xi_{\mathrm{Q} 1}=-\frac{v_{2}}{v_{1}} ; \\
& \xi_{\mathrm{Q} 2}=-\frac{v_{4}}{v_{1}} ; \\
& \xi_{\mathrm{Q} 3}=-\frac{v_{5}}{v_{1}} ; \\
& \xi_{\mathrm{Q} 4}=-\frac{v_{6}}{v_{1}} ; \\
& \xi_{\mathrm{Q} 5}=-\frac{v_{7}}{v_{1}} ; \\
& \xi_{\mathrm{Q} 6}=-\frac{v_{9}}{v_{8}} ; \\
& \xi_{\mathrm{Q} 7}=-\frac{v_{11}}{v_{8}} ; \\
& \xi_{\mathrm{Q} 8}^{2}=-\frac{v_{12}}{v_{8}} ; \\
& \omega_{\mathrm{Q} 1}^{2}=\frac{v_{3}}{v_{1}} ; \\
& v_{8}
\end{aligned}
$$

According to [20, 25-28], the solution of equation (16) can be set as

$$
\left\{\begin{array}{l}
Q_{1}=Q_{11}\left(T_{0}, T_{1}\right)+\varepsilon Q_{12}\left(T_{0}, T_{1}\right)+\mathrm{O}\left(\varepsilon^{2}\right) \\
Q_{2}=Q_{21}\left(T_{0}, T_{1}\right)+\varepsilon Q_{22}\left(T_{0}, T_{1}\right)+\mathrm{O}\left(\varepsilon^{2}\right)
\end{array}\right.
$$

where $Q_{11}$ and $Q_{21}$ are the periodic solutions of the system, $Q_{12}$ and $Q_{22}$ are the modified solutions of the system, $O\left(\varepsilon^{2}\right)$ is a higher-order small quantity, and $T_{0}$ and $T_{1}$ are two time scales $\left(T_{0}=t\right.$ and $\left.T_{1}=\varepsilon t\right)$. 


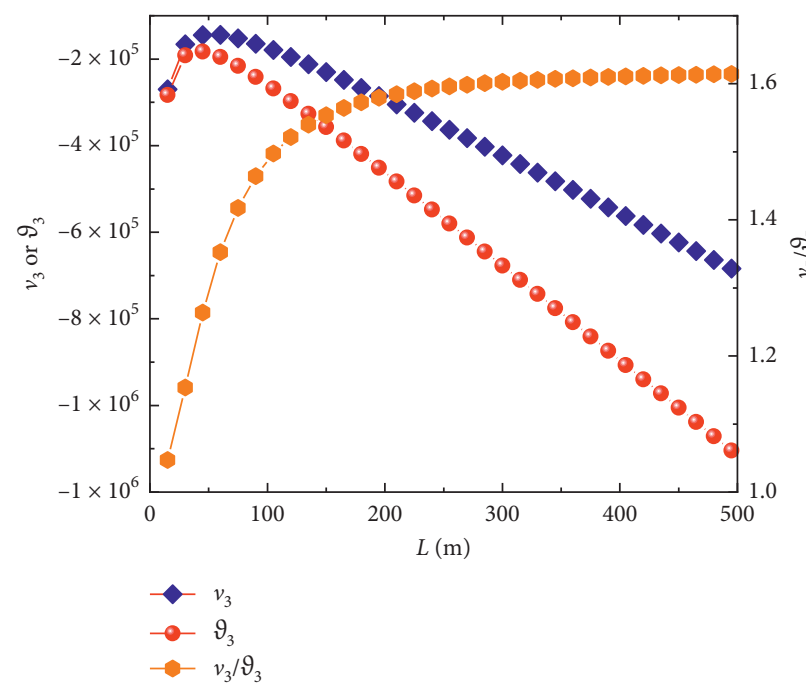

(a)

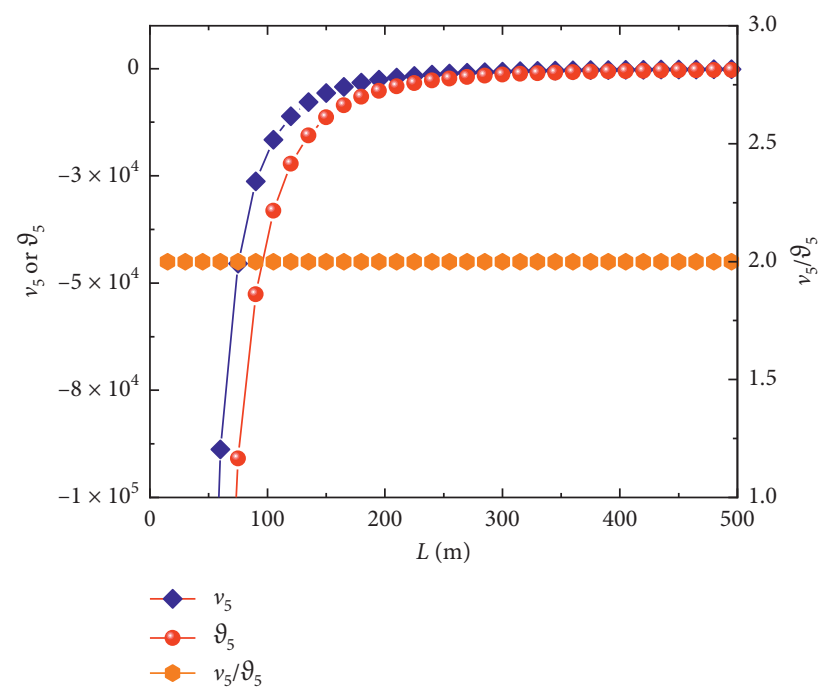

(c)

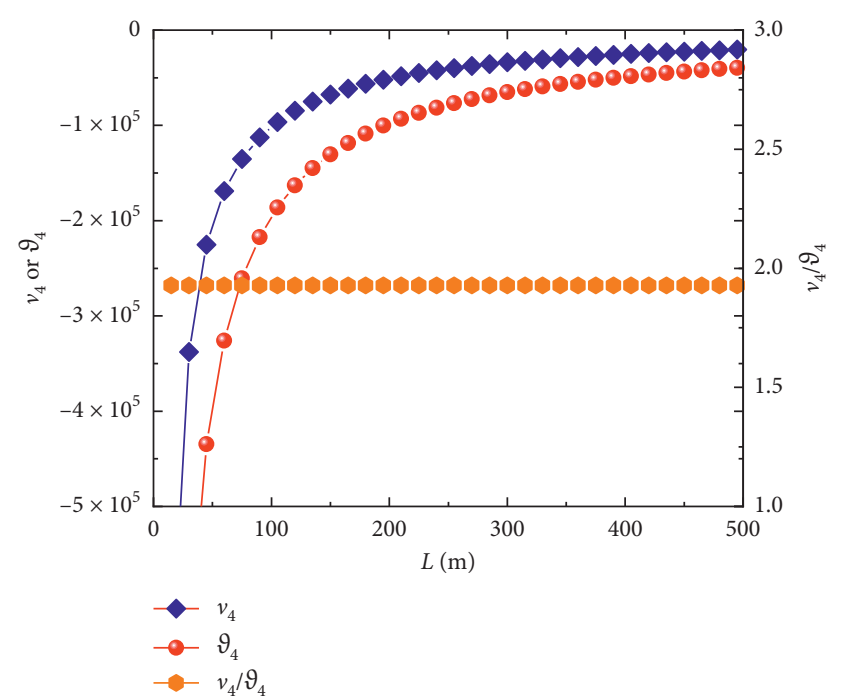

(b)

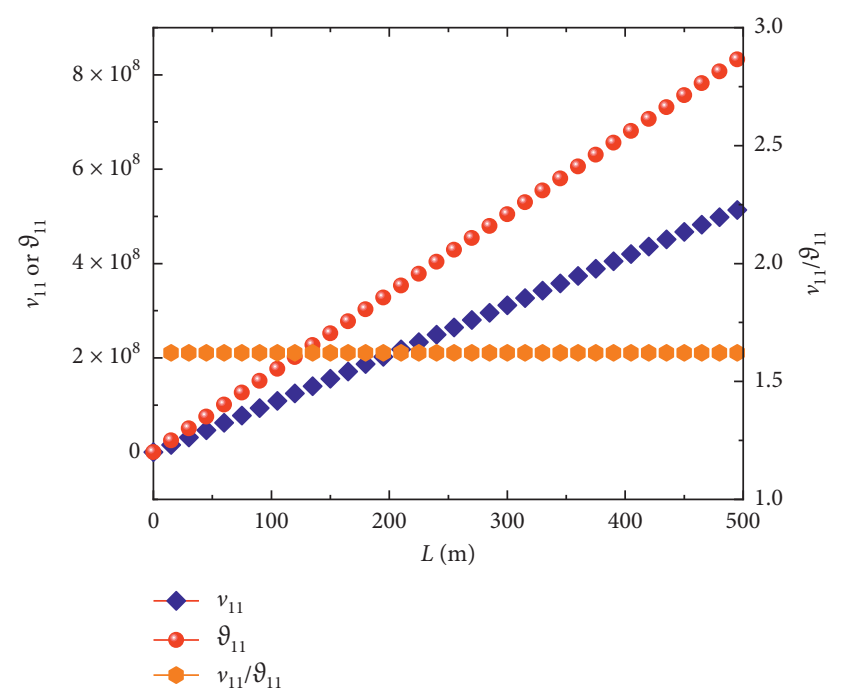

(d)

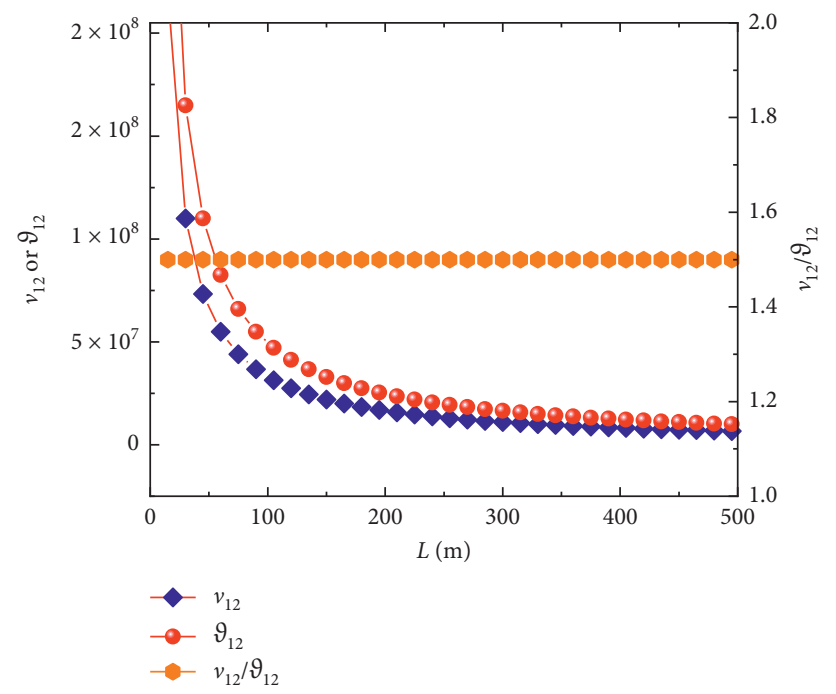

(e)

FiguRE 3: Influences of span length on coefficients: (a) coefficients of $v_{3}$ and $\vartheta_{3}$; (b) coefficients of $v_{4}$ and $\vartheta_{4}$; (c) coefficients of $\nu_{5}$ and $\vartheta_{5}$; (d) coefficients of $\nu_{11}$ and $\vartheta_{11}$; (e) coefficients of $\nu_{12}$ and $\vartheta_{12}$. 
The operator formula is introduced, which is

$$
\begin{aligned}
& D_{0}=\frac{\partial}{\partial T_{0}}, \\
& D_{1}=\frac{\partial}{\partial T_{1}} .
\end{aligned}
$$

By substituting equations (18) and (19) into equation (16) and equating the coefficients of $\varepsilon^{0}$ and $\varepsilon^{1}$ to zero independently, it can be obtained that

$\varepsilon^{0}:$

$$
\left\{\begin{array}{l}
D_{0}^{2} Q_{11}+\omega_{Q 1}^{2} Q_{11}=0 \\
D_{0}^{2} Q_{21}+\omega_{Q 2}^{2} Q_{21}=0
\end{array}\right.
$$

$\varepsilon^{1}:$

$$
\left\{\begin{array}{l}
D_{0}^{2} Q_{12}+\omega_{Q 1}^{2} Q_{12}=-2 D_{0} D_{1} Q_{11}+\xi_{\mathrm{Q} 1} D_{0} Q_{11}+\xi_{\mathrm{Q} 2} Q_{11}^{2}+\xi_{\mathrm{Q} 3} Q_{11}^{3}+\xi_{\mathrm{Q} 4} Q_{21}+\xi_{\mathrm{Q} 5} Q_{11} Q_{21} \\
D_{0}^{2} Q_{22}+\omega_{\mathrm{Q} 2}^{2} Q_{22}=-2 D_{0} D_{1} Q_{21}+\xi_{\mathrm{Q} 6} D_{0} Q_{21}+\xi_{\mathrm{Q} 7} Q_{11}+\xi_{\mathrm{Q} 8} Q_{11}^{2}
\end{array}\right.
$$

According to the solvability condition of differential equation, the solution of equation (20) can be set as follows:

$$
\left\{\begin{array}{l}
Q_{11}=A_{Q 1}\left(T_{1}\right) \exp \left(i \omega_{Q 1} T_{0}\right)+C C \\
Q_{21}=A_{Q 2}\left(T_{1}\right) \exp \left(i \omega_{Q 2} T_{0}\right)+C C
\end{array}\right.
$$

where $A_{Q 1}$ is the amplitude of the cable, $A_{Q 2}$ is the amplitude of the bridge deck, $i$ is the imaginary unit, and CC is conjugate terms.

$$
\left\{\begin{array}{l}
D_{0}^{2} Q_{12}+\omega_{\mathrm{Q} 1}^{2} \mathrm{Q}_{12}=-2 i \omega_{\mathrm{Q} 1} A_{\mathrm{Q} 1}^{\prime} \exp \left(i \omega_{\mathrm{Q} 1} T_{0}\right)+\xi_{\mathrm{Q} 1} A_{\mathrm{Q} 1} i \omega_{\mathrm{Q} 1} \exp \left(i \omega_{\mathrm{Q} 1} T_{0}\right)+ \\
\xi_{\mathrm{Q} 2}\left[A_{\mathrm{Q} 1}^{2} \exp \left(2 i \omega_{\mathrm{Q} 1} T_{0}\right)+A_{\mathrm{Q} 1} \bar{A}_{\mathrm{Q} 1}\right]+ \\
\xi_{\mathrm{Q} 3}\left[A_{\mathrm{Q} 1}^{3} \exp \left(3 i \omega_{\mathrm{Q} 1} T_{0}\right)+3 A_{\mathrm{Q} 1}^{2} \bar{A}_{\mathrm{Q} 1} \exp \left(i \omega_{\mathrm{Q} 1} T_{0}\right)\right]+ \\
\xi_{\mathrm{Q} 4} A_{\mathrm{Q} 2} \exp \left(i \omega_{\mathrm{Q} 2} T_{0}\right)+\xi_{\mathrm{Q} 5}\left\{\begin{array}{c}
A_{\mathrm{Q} 1} A_{\mathrm{Q} 2} \exp \left[i\left(\omega_{\mathrm{Q} 1}+\omega_{\mathrm{Q} 2}\right) T_{0}\right]+ \\
A_{\mathrm{Q} 1} \bar{A}_{\mathrm{Q} 2} \exp \left[i\left(\omega_{\mathrm{Q} 1}-\omega_{\mathrm{Q} 2}\right) T_{0}\right]
\end{array}\right\}+\mathrm{CC}, \\
D_{0}^{2} \mathrm{Q}_{22}+\omega_{\mathrm{Q} 2}^{2} \mathrm{Q}_{22}=-2 i \omega_{\mathrm{Q} 2} A_{\mathrm{Q} 2}^{\prime} \exp \left(i \omega_{\mathrm{Q} 2} T_{0}\right)+ \\
\xi_{\mathrm{Q} 6} A_{\mathrm{Q} 2} i \omega_{\mathrm{Q} 2} \exp \left(i \omega_{\mathrm{Q} 2} T_{0}\right)+\xi_{\mathrm{Q} 7} A_{\mathrm{Q} 1} \exp \left(i \omega_{\mathrm{Q} 1} T_{0}\right)+ \\
\xi_{\mathrm{Q} 8}\left[A_{\mathrm{Q} 1}^{2} \exp \left(2 i \omega_{\mathrm{Q} 1} T_{0}\right)+A_{\mathrm{Q} 1} \bar{A}_{\mathrm{Q} 1}\right]+\mathrm{CC},
\end{array}\right.
$$

where "' in equation (23) represents the derivation of the time scale $T_{1}$.

According to equation (23), there would be a $1: 1$ resonance mode in the cable-bridge coupling system. In the following section, the influence of the two calculation methods about dynamic tension on the resonance of the cable-bridge coupling system would be analyzed.

\section{Example Analysis}

When equivalent stiffness of bridge deck is $K=5 \times 10^{6} \mathrm{~N} / \mathrm{m}$, equivalent mass of bridge deck is $M=6.13 \times 10^{5} \mathrm{~kg}$, tension is $H=10^{5.88} \mathrm{~N}$, and span length $L$ is $300 \mathrm{~m}$. At this time, there is a $1: 1$ resonance mode in the cable-bridge coupling system under the first calculation methods of dynamic tension.

When equivalent stiffness of bridge deck is $K=5 \times 10^{6} \mathrm{~N} /$ $\mathrm{m}$, equivalent mass of bridge deck is $M=4 \times 10^{5} \mathrm{~kg}$, tension is $H=10^{5.88} \mathrm{~N}$, and span length $L$ is $300 \mathrm{~m}$. At this time, there is a $1: 1$ resonance mode in the cable-bridge coupling system under the second calculation methods of dynamic tension.

When equivalent stiffness of bridge deck is $K=5 \times 10^{6} \mathrm{~N} /$ $\mathrm{m}$, equivalent mass of bridge deck is $M=5 \times 10^{5} \mathrm{~kg}$, tension is $H=1.25 \times 10^{6} \mathrm{~N}$, and span length $L$ is $300 \mathrm{~m}$. At this time, there is no resonance mode in the cable-bridge coupling system under the two calculation methods of dynamic tension. 


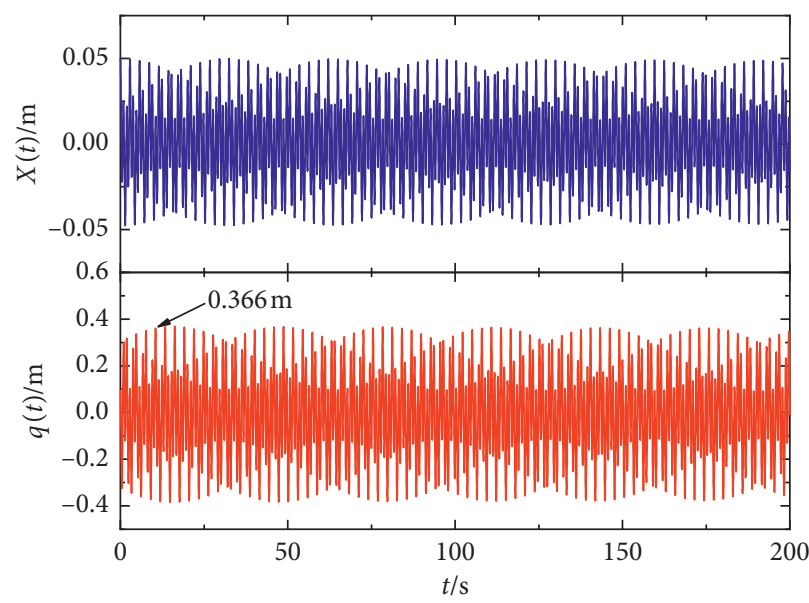

(a)

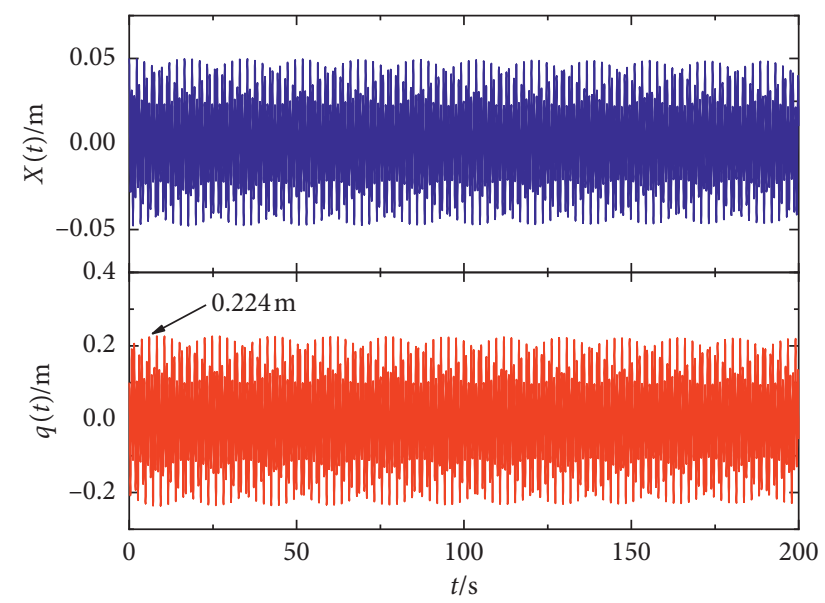

(b)

FIGURE 4: Displacement response (with 1:1 response mode): (a) the first calculation method of dynamic tension; (b) the second calculation method of dynamic tension.

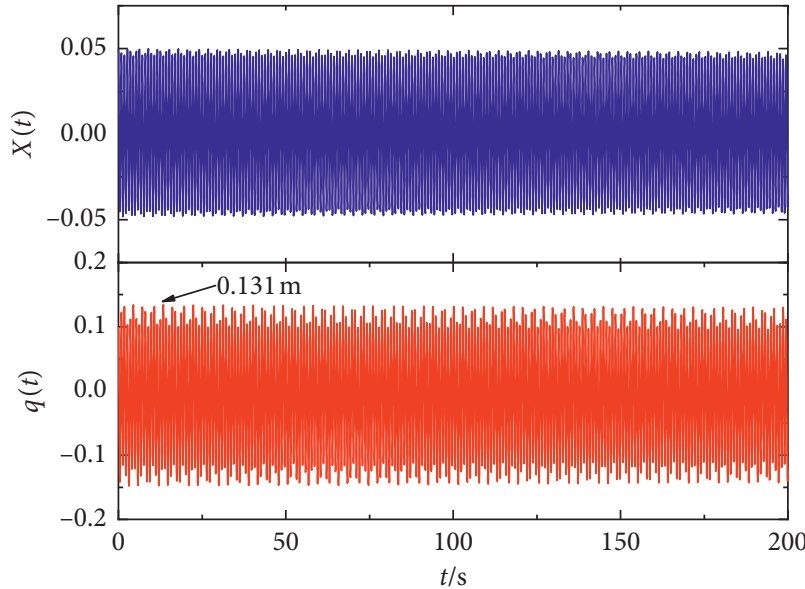

(a)

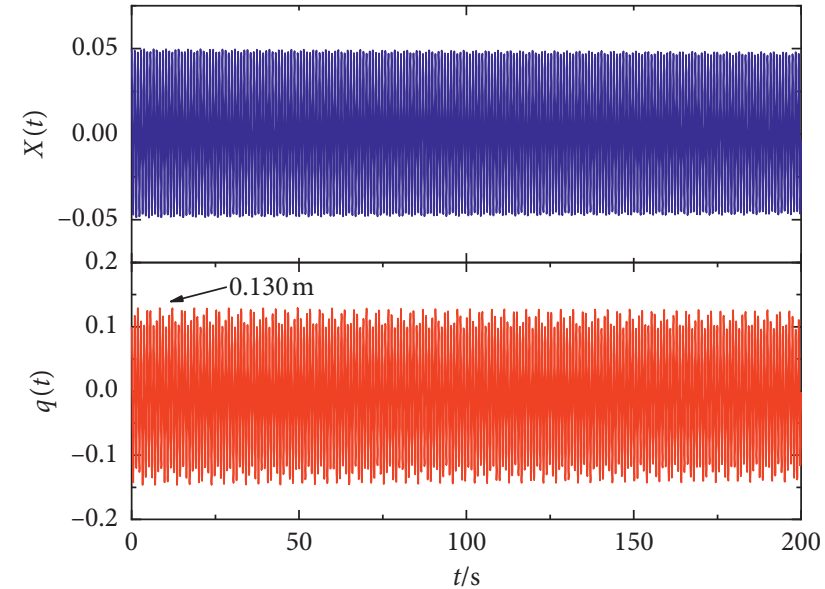

(b)

FiguRE 5: Displacement response (without 1:1 response mode): (a) the first calculation method of dynamic tension; (b) the second calculation method of dynamic tension.

Supposing that the initial displacement of the cable is $0.1 \mathrm{~m}$ and that of the bridge deck is $0.05 \mathrm{~m}$. According to Matlab, the displacement response curves of the cable-bridge coupling system under the two calculation methods of dynamic tension can be obtained, which is shown in Figures 4 and 5.

From Figure 4, it can be obtained that the beat frequency of displacement response would be different due to the differences of the calculation methods of dynamic tension. The amplitudes of the bridge deck under the two calculation methods of dynamic tension are $0.05 \mathrm{~m}$; the amplitude of the cable under the first calculation method of dynamic tension is $0.366 \mathrm{~m}$, and the amplitude of the cable under the second calculation method of dynamic tension is $0.224 \mathrm{~m}$, which is a noteworthy phenomenon. The reason for this phenomenon can be explained as follows: amplitude is a form of energy; that is, when the resonance occurs in the cable-bridge coupling system, the energy of the cable-bridge coupling system is certain, and the energy would not be different due to the different calculation methods about dynamic tension. However, the second calculation method about dynamic tension has ignored the inertial force on the cable; that is to say, it has ignored a part of energy, which would make the amplitude of the cable decrease.

From Figure 5, it can be obtained that when the cablebridge coupling system does not meet the $1: 1$ resonance condition, the displacement response curves obtained by the first calculation method about dynamic tension and the second calculation method about dynamic tension are almost the same; that is, any calculation method of dynamic tension is feasible for the nonresonance condition.

Therefore, it is reasonable to choose the first calculation method of dynamic tension to solve the displacement response of the cable due to the large energy generated by the 
resonance (the amplitude would also be large) when analyzing the resonance of the cable-bridge coupling system. For the cable-bridge coupling system without resonance, the above two calculation methods are reasonable to solve the displacement response of the cable.

\section{Conclusion}

Based on the theoretical analysis and numerical simulation, the influences of the two calculation methods about dynamic tension of cables on the linear coefficient and nonlinear coefficient in the ordinary differential vibration equation are analyzed. Also, the displacement response of the cablebridge coupling model under the two calculation methods about dynamic tension is compared. The following conclusions can be listed:

(1) There are obvious differences in some coefficients in the ordinary differential vibration equation of the cable-bridge coupling model obtained by the two calculation methods about dynamic tension. Because the vibration frequency of the cable is dominated by coefficients $v_{3}$ and $\vartheta_{3}$, the differences of coefficients $\nu_{3}$ and $\vartheta_{3}$ would make the vibration characteristics of cable-bridge coupling model be different.

(2) When the $L$ is greater than $300 \mathrm{~m}$, the ratios of coefficients obtained by these two calculation methods all are certain values which range from 1.5 to 2.0; while when the $L$ is less than $300 \mathrm{~m}$, the ratio of coefficients $\nu_{3}$ and $\vartheta_{3}$ is a change value, which increases with the increasing $L$ and finally tends to be 1.62 .

(3) The energy of the cable-bridge coupling model is certain, and the energy would not be different due to the different calculation methods about dynamic tension. However, the second calculation method about dynamic tension has ignored the inertial force on the cable; that is to say, a part of the energy is ignored, which would reduce the amplitude of the cable.

(4) For the cable-bridge coupling model with $1: 1$ resonance mode, because the energy generated by $1: 1$ resonance is large (the amplitude of cables would be large), it is reasonable to choose the first calculation method of dynamic tension to solve the displacement response of the cable.

(5) For the cable-bridge coupling model without a resonance mode, because the energy generated by nonresonance mode is small (the amplitude of cables would be small), these two calculation methods about dynamic tension both are reasonable to solve the displacement response of the cable.

The influence of two calculation methods about dynamic tension on the vibration characteristics of the cable-bridge coupling model has been considered in this paper, and then, it can be found that the conclusions here can be helpful to the perfection of the cable-bridge coupling model and have some guiding value for practical engineering.

\section{Data Availability}

The data used to support this study are available from the corresponding author upon request.

\section{Conflicts of Interest}

The authors declare that there are no conflicts of interest regarding the publication of this paper.

\section{Acknowledgments}

This work was financially supported by the National Natural Science Foundation of China (51507106 and 51308570), Cutting Edge Project of Chongqing Science and Technology Commission (cstc2017jcyjAX0246), Graduate Research and Innovation Project of Chongqing (cys19240), China Postdoctoral Science Foundation (2020M672238), Chengdu International Science and Technology Cooperation Support Funding (2020-GH02-00059-HZ), and Technology Research Project of Chongqing Education Commission (KJ201600712182).

\section{References}

[1] W. P. Randall, "Cable-stay conundrum," Civil Engineering, vol. 31, no. 1, pp. 58-61, 1998.

[2] B. Asgari, S. A. Osman, and A. Adnan, "A new multiconstraint method for determining the optimal cable stresses in cablestayed bridges," The Scientific World Journal, vol. 2014, Article ID 503016, 19 pages, 2014.

[3] G. Tagata, "Harmonically forced, finite amplitude vibration of a string," Journal of Sound and Vibration, vol. 51, no. 4, pp. 483-492, 1977.

[4] G. You, "A structural analysis method for cable-beam composite structure," Mathematical Problems in Engineering, vol. 2020, no. 4, 13 pages, 2020.

[5] Y. Cong, H. Kang, and T. D. Guo, "Planar multimodal $1: 2: 2$ internal resonance analysis of cable-stayed bridge," $\mathrm{Me}$ chanical Systems and Signal Processing, vol. 120, no. 2019, pp. 505-523, 2019.

[6] K. Wilde and W. Witkowski, "Simple model of rain-windinduced vibrations of stayed cables," Journal of Wind Engineering and Industrial Aerodynamics, vol. 91, no. 7, pp. 873-891, 2003.

[7] J. Peng, M. Xiang, L. Li, H. Sun, and X. Wang, “Time-delayed feedback control of piezoelectric elastic beams under superharmonic and subharmonic excitations," Applied Sciences, vol. 9, no. 8, p. 1557, 2019.

[8] Z. Kang and W. X. Zhong, "Numerical study on parametric vibration of cable in cable stayed bridge," China Civil Engineering Journal, vol. 31, no. 4, pp. 3-5, 1998.

[9] Z. G. Wang and B. N. Sun, "Cable Vibration for cable stayed bridge by parametric response," Engineering Mechanics, vol. 18, no. 1, pp. 103-109, 2001, in Chinese.

[10] S. S. Chen and B. N. Sun, "Numerical study on nonlinear parametric vibration of coupled cables and bridge decks," China Civil Engineering Journal, vol. 36, no. 4, pp. 70-75, 2003, in Chinese.

[11] Y. Y. Zhao, T. Wang, H. J. Kang, and L. H. Wang, "Performance study of the nonlinear parametric vibration of coupled bridge decks and two cables," Journal of Hunan University (Natural Sciences), vol. 35, no. 10, pp. 1-5, 2008, in Chinese. 
[12] Z. G. Zhou, F. Wang, and L. H. Deng, "Geometrically nonlinear analysis of composite girder cable-stayed bridge with three towers in completion stage," Applied Mechanics and Materials, vol. 166-169, pp. 2761-2764, 2012.

[13] M. H. Wei, K. Lin, L. Jin, and D. J. Zou, "Nonlinear dynamics of a cable-stayed beam driven by sub-harmonic and principal parametric resonance," International Journal of Mechanical Sciences, vol. 110, no. 2016, pp. 78-93, 2016.

[14] Y. Zhao, Z. Wang, X. Zhang, and L. Chen, "Effects of temperature variation on vibration of a cable-stayed beam," International Journal of Structural Stability and Dynamics, vol. 17, no. 10, Article ID 1750123, 2017.

[15] M. Lepidi, V. Gattulli, and F. Vestroni, "Static and dynamic response of elastic suspended cables with damage," International Journal of Solids and Structures, vol. 44, no. 25-26, pp. 8194-8212, 2007.

[16] Y. Zhao, J. Peng, Y. Zhao, and L. Chen, "Effects of temperature variations on nonlinear planar free and forced oscillations at primary resonances of suspended cables," Nonlinear Dynamics, vol. 89, no. 4, pp. 2815-2827, 2017.

[17] C. Sun, Y. Zhao, J. Peng, H. Kang, and Y. Zhao, "Multiple internal resonances and modal interaction processes of a cable-stayed bridge physical model subjected to an invariant single-excitation," Engineering Structures, vol. 172, no. 172, pp. 938-955, 2018.

[18] Q. X. Wu, Y. C. Kitahara, K. Takahashi, and B. C. Chen, "Dynamic characteristics of Megami cable-stayed bridge-a comparison of experimental and analytical results," International Journal of Steel Structures, vol. 8, no. 1, pp. 1-9, 2008.

[19] A. Luongo, D. Zulli, and G. Piccardo, "Analytical and numerical approaches to nonlinear galloping of internally resonant suspended cables," Journal of Sound and Vibration, vol. 315, no. 3, pp. 375-393, 2008.

[20] X. Liu, G. Min, C. Wu, M. Cai, and C. Wu, "Investigation on influences of two discrete methods on galloping characteristics of iced quad bundle conductors," Advances in Civil Engineering, vol. 2020, Article ID 8818728, 17 pages, 2020.

[21] S. Refrafi, A. A. Bousahla, A. Bouhadra et al., "Effects of hygrothermo-mechanical conditions on the buckling of FG sandwich plates resting on elastic foundations," Computers and Concrete, vol. 25, no. 4, pp. 311-325, 2020.

[22] M. Rabhi, K. H. Benrahou, A. Kaci et al., "A new innovative 3-unknowns HSDT for buckling and free vibration of exponentially graded sandwich plates resting on elastic foundations under various boundary conditions," Geomechanics and Engineering, vol. 22, no. 2, pp. 119-132, 2020.

[23] A. Zine, A. A. Bousahla, F. Bourada et al., "Bending analysis of functionally graded porous plates via a refined shear deformation theory," Computers and Concrete, vol. 26, no. 1, pp. $63-74,2020$.

[24] A. Menasria, A. Kaci, A. A. Bousahla et al., "A four-unknown refined plate theory for dynamic analysis of FG-sandwich plates under various boundary conditions," Steel and Composite Structures, vol. 36, no. 3, pp. 355-367, 2020.

[25] N. C. Perkins, "Modal interactions in the non-linear response of elastic cables under parametric/external excitation," International Journal of Non-linear Mechanics, vol. 27, no. 2, pp. 233-250, 1992.

[26] L.-N. Zhang, F.-C. Li, X.-Y. Wang, and P.-F. Cui, "Theoretical and numerical analysis of $1: 1$ main parametric resonance of stayed cable considering cable-beam coupling," Advances in Materials Science and Engineering, vol. 2017, Article ID 6948081, 10 pages, 2017.
[27] Y. Zhao, C. Sun, Z. Wang, and L. Wang, "Analytical solutions for resonant response of suspended cables subjected to external excitation," Nonlinear Dynamics, vol. 78, no. 2, pp. 1017-1032, 2014.

[28] X. Liu, G. Min, C. Sun, and M. Cai, "Investigation on stability and galloping characteristics of iced quad bundle conductor," Journal of Applied Fluid Mechanics, vol. 14, no. 1, pp. 117-129, 2021. 